\title{
Clinical courses and degradation patterns of absorbable plates in facial bone fracture patients
}

\author{
Young Min Kim, \\ Jong Hun Lee \\ Department of Plastic and \\ Reconstructive Surgery, Nowon Eulji \\ Medical Center, Eulji University School \\ of Medicine, Seoul, Korea
}

\begin{abstract}
Background: Absorbable plates are widely used in open reduction and internal fixation surgeries for facial bone fractures. Absorbable plates are made of polyglycolic acid (PGA), polylactic acid (PLA), polydioxane (PDS), or various combinations of these polymers. The degradation patterns of absorbable plates made from different polymers and clinical courses of patients treated with such plates have not been fully identified. This study aimed to confirm the clinical courses of facial bone fracture patients using absorbable plates and compare the degradation patterns of the plates.

Methods: A retrospective chart review was conducted for 47 cases in 46 patients who underwent open reduction and internal fixation surgery using absorbable plates to repair facial bone fractures. All surgeries used either PLA/PGA composite-based or poly-L-lactic acid (PLLA)/hydroxyapatite (HA) composite-based absorbable plates and screws. Clinical courses were confirmed and comparisons were conducted based on direct observation.

Results: There were no naturally occurring foreign body reactions. Post-traumatic inflammatory responses occurred in eight patients (nine cases), in which six recovered naturally with conservative treatment. The absorbable plates were removed from two patients. PLA/PGA compositebased absorbable plates degraded into fragments with non-uniform, sharp surfaces whereas PLLA/HA composite-based absorbable plates degraded into a soft powder.

Conclusion: PLA/PGA composite-based and PLLA/HA composite-based absorbable plates showed no naturally occurring foreign body reactions and showed different degradation patterns. The absorbable plate used for facial bone fracture surgery needs to be selected in consideration of its degradation patterns.
\end{abstract}

Keywords: Absorbable implants / Bone plates / Fractures / Hydroxyapatites / Polyglycolic acid

\section{INTRODUCTION}

Titanium plates and absorbable plates are being widely used in

\section{Correspondence: Jong Hun Lee}

Department of Plastic and Reconstructive Surgery, Nowon Eulji Medical Center, Eulji University School of Medicine, 68 Hangeulbiseong-ro, Nowon-gu, Seoul 01830, Korea

E-mail: joaljh@eulji.ac.kr

${ }^{*}$ This article was presented at the 7th Research \& Reconstruction Forum on April 20, 2017, in Daejeon, Korea.

Received August 27, 2019 / Revised October 16, 2019 / Accepted October 18, 2019 open reduction and internal fixation surgeries for facial bone fractures. Titanium metal-based plates provide rigid fixation; however, their main drawbacks include stress-shielding, bone atrophy from device corrosion, distortions on radiological images, and the need for secondary surgery to remove the device. In contrast, absorbable plates, despite their relatively high cost, provide weaker fixation due to biodegradation over time, which promotes bone regeneration and allows for cranial or facial bone growth in children [1-4].

In general, absorbable plates used for fixation of fractures are 
created using polyglycolic acid (PGA), polylactic acid (PLA), polydioxane (PDS), or combinations of these materials. PGA has a crystalline structure and thus is stronger than PLA; however, its strength diminishes within 6 weeks and the material is quickly absorbed within 3-12 months. PLA is hydrophobic; it therefore takes up to 5 years for the material to be absorbed $[5,6]$. There are two types of commercialized, widely used absorbable plates: polymers of PLA or PGA, or polymers of polyL-lactic acid (PLLA)/hydroxyapatite (HA). The PLA/PGA composite-based polymers have exceptional flexibility and biocompatibility. These plates are degraded within 9-15 months after the procedure and the pieces are absorbed by macrophages and giant cells [5,7]. PLLA/HA composite-based polymers are manufactured by polymerizing PLLA with the inorganic compound HA, which constitutes calcified tissue. The resulting product contains non-calcified, unsintered $\mathrm{HA}\left(\mathrm{Ca}_{10}\right.$ $\left.\left(\mathrm{PO}_{4}\right)_{6}(\mathrm{OH})_{2}\right)$, which makes it bioactive and bioresorbable. Additionally, it exhibits exceptional cohesion and strength, a rapid biodegradation rate, and relatively uniform-sized degradation particles compared to PLLA alone [8-11].

However, there is limited research on the clinical courses of patients who underwent surgeries using absorbable plates. Moreover, the degradation patterns of absorbable plates made from different materials have only been observed in animal studies; how absorbable plates degrade in humans remains unclear [12]. Therefore, this study aimed to confirm the clinical courses of patients who underwent reconstruction of facial bone fractures using absorbable plates and to compare the degradation patterns of two types of absorbable plates made from different materials in patients who underwent open reduction and internal fixation surgeries for facial bone fractures.

\section{METHODS}

\section{Study design}

We conducted a retrospective medical chart review of 47 cases in 46 patients who underwent open reduction and internal fixation for zygomaticomaxillary fractures using absorbable plates at our institution between January 2010 and April 2016. A single surgeon performed the open reduction and internal fixation surgeries in all the patients. The study was approved by the appropriate institutional review board and informed consent was obtained from all the patients. The study design followed the guidelines of the 1975 Declaration of Helsinki. Surgeries were performed within 2 weeks of the injury. Preoperative physical examinations, radiological examinations, and computed tomography (CT) scans were performed to diagnose the fracture and establish the surgical plan.

\section{Surgical procedures}

All surgeries were performed under general anesthesia. Both intraoral and subciliary incisions were made to expose the fracture site and to confirm the location of the displaced bone fragments. After reduction of the displaced bone fragments to their original anatomical position, their positions relative to nearby bone structures were confirmed. Absorbable plates and screws were used to achieve fixation and the two incised areas were closed after sterile irrigation. For intraoral incisions, 4-0 vicryl was used for suturing whereas 6-0 nylon was used for suturing subciliary incisions.

\section{Long-term evaluation}

After an average of 5 days of postoperative hospitalization, the patients continued to receive outpatient treatment at our institution. The follow-up period was between 1 month and 5 years, with a mean duration of 27.5 months.

\section{Properties of products}

PLA/PGA composite-based plates (Inion CPS; Inion Co., Ltd., Tampere, Finland) or PLLA/HA composite-based plates (Osteotrans Mx; Takiron Co., Ltd., Hyogo, Japan) were used for the fixation of the fractured facial bones.

\section{RESULTS}

Among the 47 cases in 46 patients who underwent the procedure, PLA/PGA and PLLA/HA composite-based absorbable plates were used in 32 and 15 cases, respectively. Both types of implants were used on one case which sustained zygomaticomaxillary fractures at different times on different sides of the face. The patients' age distribution ranged from 11 to 65 years, with a mean age of 31.3 years. Of the 46 patients, 38 were male and eight were female. There were 13 injuries from slips, 12 from sports, nine from traffic accidents, five from violence, two from falls, and six from other contusions. Postoperative X-ray images confirmed adequate reduction of fractures without displacement of bone fragments and all patients were discharged without notable issues. There were no complications (i.e., hematoma or infection) immediately after surgery and all patients were followed-up between 1 month and 5 years.

No patient had naturally occurring foreign body reactions, such as swelling or erythematous reactions, within 6 months of surgery. This suggests that both types of plates are biocompatible. However, there were nine traumatic injuries around the surgical site and these patients had post-traumatic inflammatory responses. Among them, six had undergone surgery using a PLA/PGA composite-based polymer while the other three un- 
derwent surgery using a PLLA/HA composite-based polymer. Six cases recovered after conservative treatment, such as cold therapy and nonsteroidal anti-inflammatory drugs (Table 1).

In the three cases in two patients who underwent secondary surgeries to remove the fixation device because of continuous post-traumatic inflammatory responses, we compared the visible differences between the degradation patterns of the fixation materials. A PLA/PGA composite-based absorbable plate was used in one of these patients and was removed 14 months after reduction. In the other patient, a PLA/PGA composite-based absorbable plate was used on the left side of the face while a PLLA/HA composite-based absorbable plate was used on the right side of the face at different time points, with each being removed 24 months after reduction (Table 1 ).

One of these two patients underwent sequential surgeries using PLA/PGA composite-based polymers first then using PLLA/HA composite-based polymers for bilateral zygomaticomaxillary fractures that occurred at different times. The patient required removal of both plates and differences were observed in the appearance of the degraded material removed from each side. This case allowed for direct comparison of the degradation patterns of absorbable plates made from different materials in the same person.

This patient visited our center with a right zygomaticomaxillary fracture 2 years after the initial left side fracture. For the initial zygomaticomaxillary fracture surgery on the left side, the patient did not want foreign substances to remain inside their body and therefore underwent surgery using an absorbable PLA/PGA polymer-based plate instead of a metal-based plate (Fig. 1).

The patient did not exhibit any noticeable abnormalities after the surgery until 2 years later when swelling and redness became persistent on the left side of his face. The patient was diagnosed with post-traumatic inflammatory response to a foreign body (Fig. 2). The PLA/PGA polymer-based absorbable plate was surgically removed. The partially degraded absorb-

Table 1. Data on post-traumatic inflammatory cases

\begin{tabular}{|c|c|c|c|c|c|c|}
\hline Case & Age $(y r) / s e x$ & Fracture site & Inflammatory sign & Plate type & Treatment & Result \\
\hline 1 & 36/Male & Rt. ZMC & Painful swelling & PLA/PGA & NSAID & Recovered \\
\hline 2 & 25/Female & Rt. ZMC & Redness, Tenderness & PLLA/HA & NSAID, cooling & Recovered \\
\hline 3 & 21/Male & Lt. ZMC & Redness, Swelling & PLA/PGA & NSAID, cooling & Recovered \\
\hline 4 & 18/Male & Rt. ZMC & Painful swelling & PLA/PGA & NSAID & Recovered \\
\hline 5 & 42/Female & Rt. ZMC & Redness, Swelling & PLA/PGA & NSAID & Recovered \\
\hline 6 & 47/Male & Lt. ZMC & Redness, Tenderness & PLLA/HA & NSAID, cooling & Recovered \\
\hline 7 & 56/Male & Rt. ZMC & Redness, Tenderness & PLA/PGA & Implant removal & Recovered \\
\hline $8^{\text {a) }}$ & 26/Male & Lt. ZMC & Redness, Tenderness & PLA/PGA & Implant removal & Recovered \\
\hline 9) & 26/Male & Rt. ZMC & Redness, Tenderness & PLLA/HA & Implant removal & Recovered \\
\hline
\end{tabular}

Rt, right; Lt, left; ZMC, zygomaticomaxillary complex; PLA, polylactic acid; PGA, polyglycolic acid; NSAID, nonsteroidal anti-inflammatory drugs; HA, hydroxyapatite. ${ }^{a}$ Cases 8 and 9 are the same person who experienced bilateral ZMC fractures at different times.
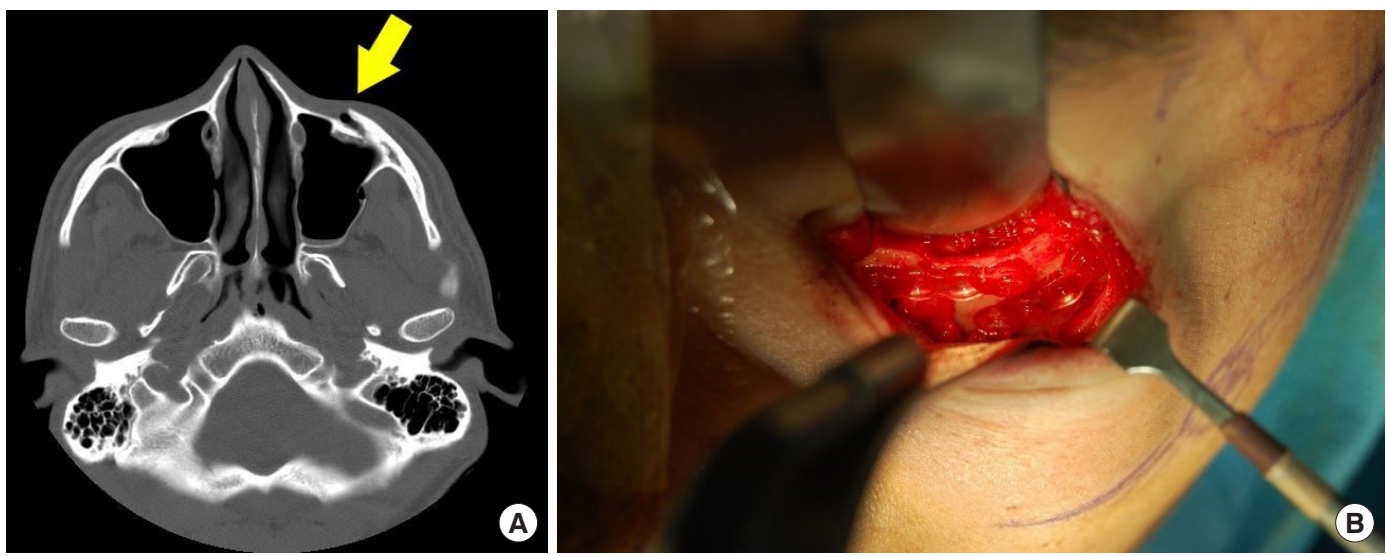

Fig. 1. A 26-year-old man with a zygomaticomaxillary fracture on his left side. He underwent an operation using PLA/PGA composite-based absorbable plates and screws. (A) Preoperative computed tomography scans demonstrating maxilla fracture with anterior wall dislocation (arrow). (B) Intraoperative photograph. Fractured left maxilla anterior wall fixed using PLA/PGA composite-based absorbable plates and screws. PLA, polylactic acid; PGA, polyglycolic acid. 

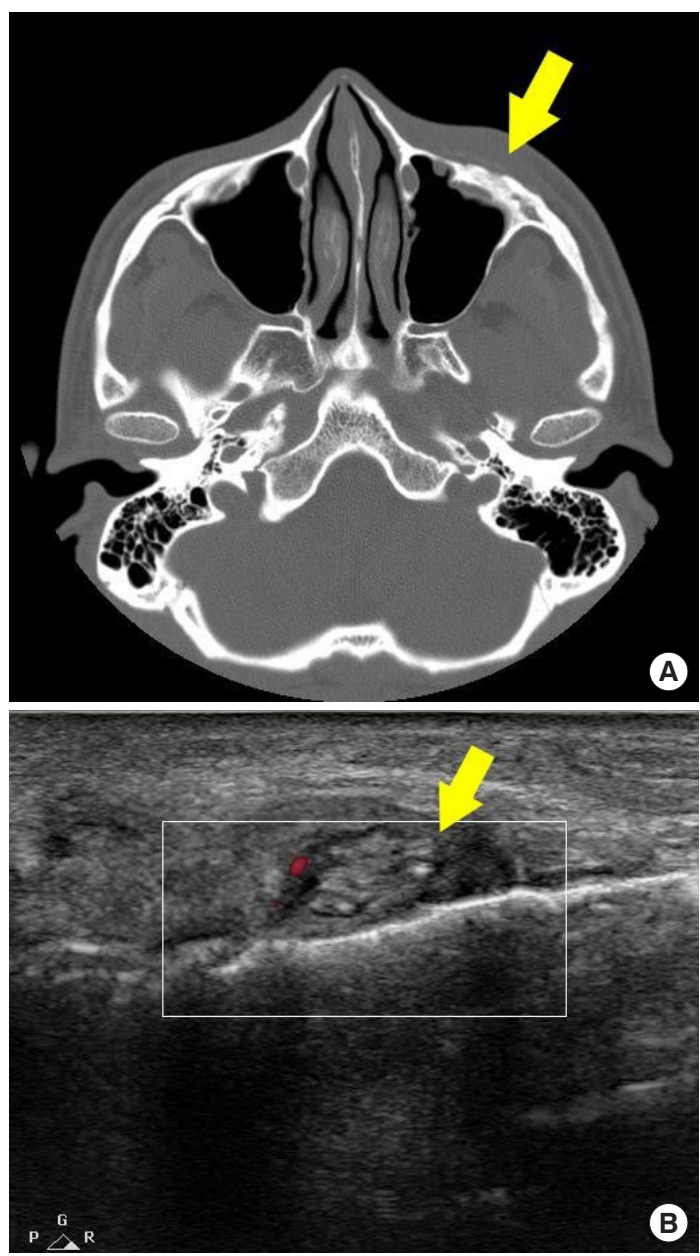

Fig. 2. Post-traumatic inflammatory response at 24 months after the initial left maxilla anterior wall reduction surgery using PLA/ PGA absorbable plates and screws. (A) Postoperative computed tomography image at 24 months showing rough and uneven surface on the left maxilla anterior wall. Mild left cheek soft tissue swelling is seen (arrow). (B) Postoperative ultrasound finding at 24 months showing multiple tiny materials that formed a cloud-like mass (arrow). PLA, polylactic acid; PGA, polyglycolic acid.

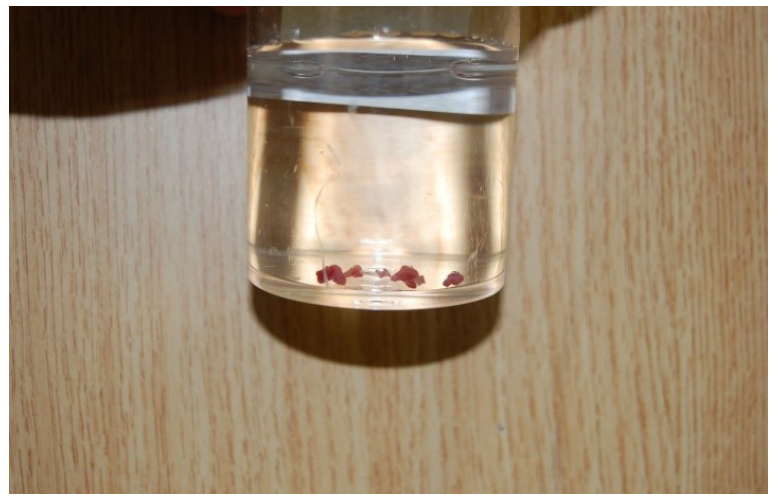

Fig. 3. Foreign body material removed from the left maxilla anterior wall during reduction surgery using PLA/PGA absorbable plates and screws. Solid and acute tiny materials were found. PLA, polylactic acid; PGA, polyglycolic acid.
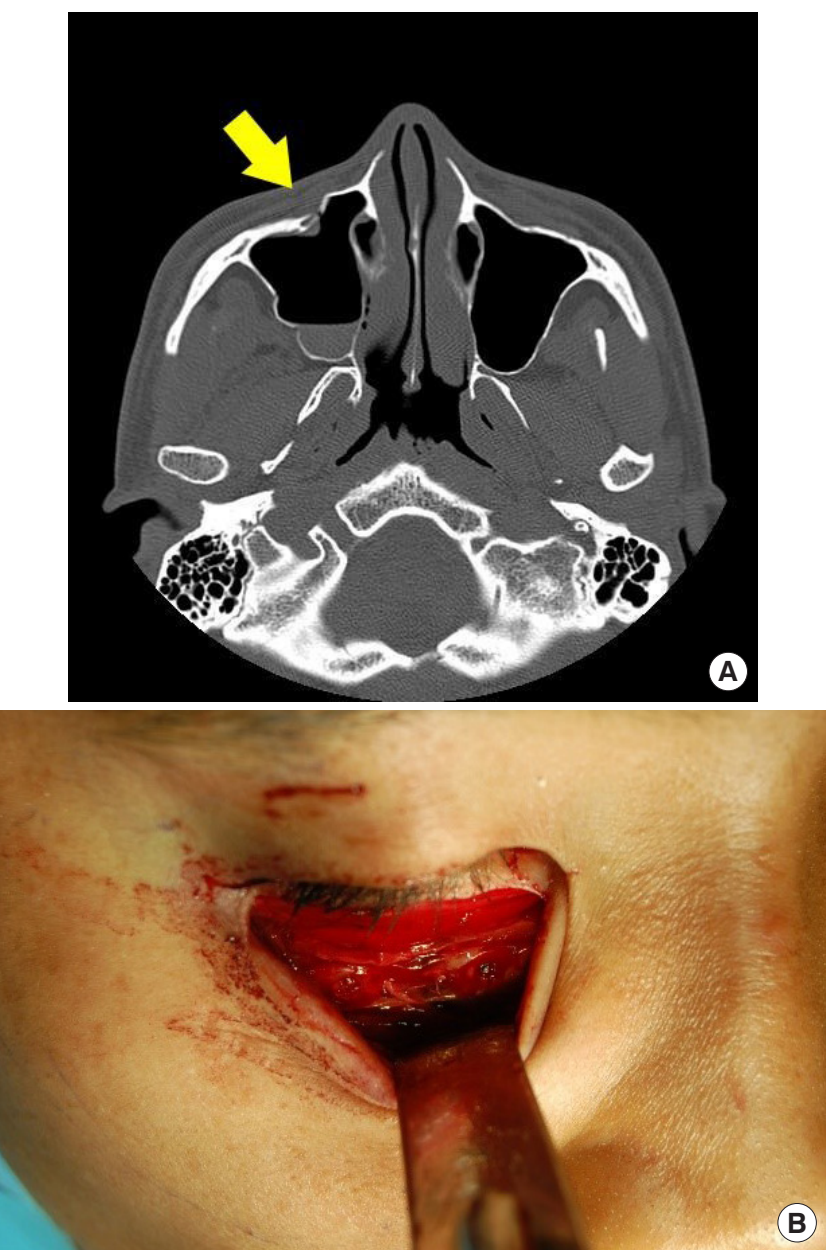

Fig. 4. A 26-year-old man (same as in Fig. 1) had additional surgery due to a zygomaticomaxillary fracture on the right side. He underwent an operation using PLLA/HA composite-based absorbable plates and screws. (A) Preoperative computed tomography scans demonstrating right maxilla fracture with anterior wall dislocation (arrow). (B) Intraoperative photograph. Fractured right maxilla anterior wall fixed using PLLA/HA absorbable plate and screws. PLLA, poly-L-lactic acid; HA, hydroxyapatite.

able plate was solid and contained sharp fragments (Fig. 3). After removal of the plate, the injury healed without additional complications.

Two months after removal of the PLA/PGA polymer-based absorbable plate, the patient visited our center with a zygomaticomaxillary fracture on the right side and underwent additional surgery. The patient requested that a different type of absorbable plate be used; therefore, a PLLA/HA polymer-based absorbable material was used in this surgery (Fig. 4).

There were no notable abnormalities until 2 years after the surgery when the patient was diagnosed with inflammatory response to a foreign body based on persistent swelling and redness on his right cheek after sustaining a traumatic injury to his right cheek. The partially degraded PLLA/HA polymer-based 

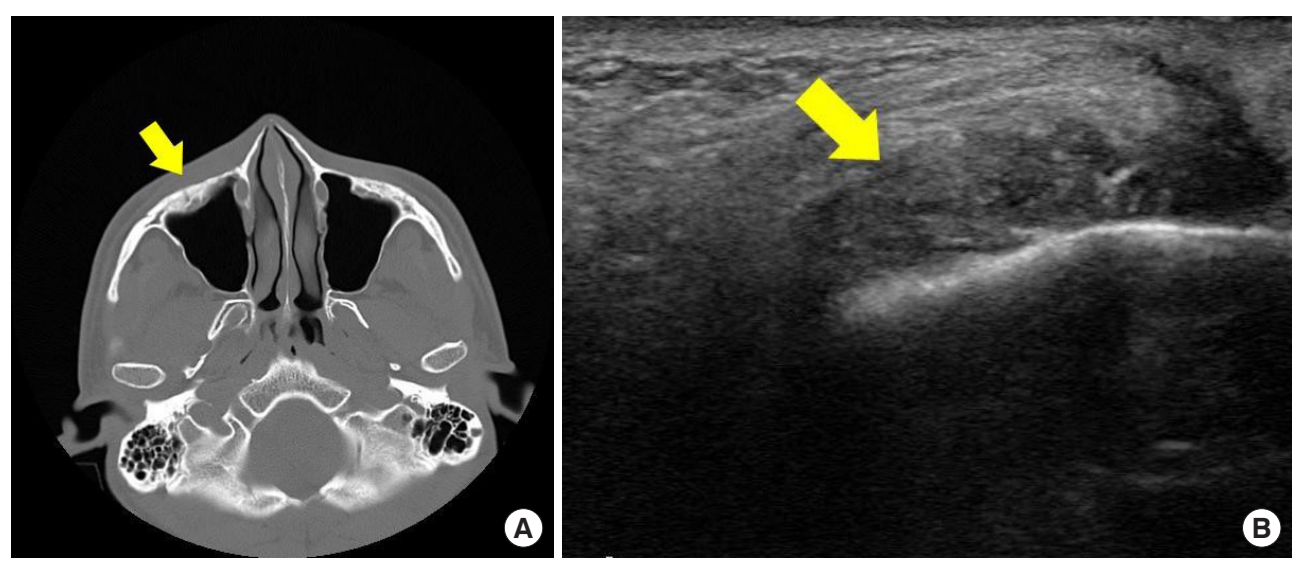

Fig. 5. Post-traumatic inflammatory response at 24 months after the right maxilla anterior wall reduction surgery using PLLA/HA absorbable plates and screws. (A) Postoperative computed tomography image at 24 months showing soft and even surface on the right maxilla anterior wall (arrow). (B) Postoperative ultrasound finding at 24 months showing powder-like foreign materials scattered around the surgery site (arrow). PLLA, poly-L-lactic acid; HA, hydroxyapatite.

absorbable plate was surgically removed. Unlike the PLA/PGA plate, what remained of the PLLA/HA plate was small powderlike particles that were removed by irrigation (Fig. 5). After removal of the remnants of the degraded plate, the injury healed without further complications.

\section{DISCUSSION}

Many surgeries are performed to correct facial bone fractures for various reasons, including traumatic injury or aesthetic purposes. For facial bones, both surgeons and patients have more realistic expectations compared to surgeries on upper or lower limbs. Therefore, different implant materials are selected for fixation of bone fragments in limbs versus faces. For fixation of bone fractures in upper and lower limbs, titanium-based plates are often used; however, an additional surgery is required after synostosis to remove the metal and prevent retained titanium. In contrast, for fixation of facial bone fractures, there is an increasing use of plates made from absorbable materials, negating the need for secondary surgeries. Consequently, various types of absorbable materials are being widely used to make plates for fixation of facial bone fractures. The major reason for using plates made of absorbable materials for fixation of facial bone fractures is that there would be no remaining foreign material in the body after biodegradation given a sufficient period of time. Therefore, understanding the degradation patterns of absorbable plates made from different materials is crucial.

The crystallinity of the material used in absorbable plates has the greatest impact on its absorption and degradation. Materials with higher crystallinity result in greater amounts of crystalline material formed, are more resistant to degradation and absorption, and remain in the body for a longer period of time, which could induce more tissue-level responses like foreign body reactions, compared to materials with lower crystallinity. In rare circumstances, these tissue-level reactions could include inflammation, swelling, acute infection, and cyst formation. In contrast, materials with lower crystallinity and smaller volumes of crystalline material formed correlate with faster degradation and absorption. Other factors that affect the degradation and absorption of materials used in absorbable plates include the size and shape of the plates, circulation, thickness of the soft tissue, and the transplant site. More specifically, smaller plate sizes are associated with faster degradation and absorption [13-15].

Degradation of PLA/PGA composite-based polymers begins with the breakage of the polymer chain via hydrolysis, resulting in smaller fragments. First, the molecular weight of the plate becomes smaller and the reduced coherence results in easier absorption of the material as it is shredded into smaller pieces. Subsequently, the products generated by hydrolysis undergo phagocytosis and intracellular absorption by macrophages and tissue cells, where they are converted into $\mathrm{CO}_{2}$ and $\mathrm{H}_{2} \mathrm{O}$ through the tricarboxylic acid or Krebs cycle and are eliminated from the body through the lungs and kidneys. The balance between hydrolysis and metabolism is crucial; if the amount of products generated by hydrolysis exceeds the amount eliminated by metabolism, inflammation occurs. Degraded absorbable plate material consisting of excessive products generated by hydrolysis may act as culture medium for bacteria [16-19].

Degradation of PLLA/HA composite-based polymers requires progressive loss of coherence, absorption of PLLA once degraded into oligomers, and discharge of uncombined HA debris and calcium phosphate deposits. Once fixed to the bone, the PLLA portion of PLLA/HA composite-based polymers hydrolyzes with body fluids. In general, plates consistently release 
uncombined HA molecules for the first 2.5 years, causing the plates to gradually decrease in size. Up to $2-2.5$ years after the insertion of the absorbable plate, the proportion of PLLA dramatically decreases and the fraction of HA in the polymer increases. After 4-5 years, the PLLA portion of the polymer is completely gone. Almost all HA molecules are replaced with bone structures after 5.5 years [10]. Because no chemical bonds are formed between PLLA and HA and they have permeable surfaces, body fluids easily diffuse into them. Therefore, these polymers degrade more quickly than PLLA polymers. In addition, unsintered HA molecules in the PLLA/HA compositebased polymers have low crystallinity, making them more easily absorbable. Because these molecules generally degrade through chemical dissolution rather than phagocytosis, inflammatory cells are not involved. Therefore, HA molecules have a lower possibility of inducing tissue-level responses than PLLA molecules $[10,20]$.

In our study, the degradation patterns observed were not in agreement with results of previous studies that used animal models. For PLA/PGA composite-based plates that are composed of only polymers, previous studies reported that these plates generally undergo complete degradation; however, we observed remaining fragments near the surgery site 2 years after the operation [12]. In our study, we observed PLLA/HA composite-based plates that degraded into uniform, powderlike particles over time. Because these plates provide adequate strength throughout the period of synostosis and degrade into uniform, powder-like particles, they may be ideal materials for absorbable plates used in facial bone surgeries.

Previous studies have shown that PLA/PGA composite-based plates degrade faster than PLLA/HA composite-based plates. Direct visual confirmation of this study revealed that of the two plates removed at the same postoperative time point, PLLA/HA was more absorbent and was observed to remain as fine powder. The clinical significance of this study is the direct observation and comparison of the removal of absorbable plates in the human body, not in laboratory tests or animal experiments.

There were no spontaneous foreign body reactions in 38 of the 46 patients. Eight patients had traumatic injuries at the surgical site. This suggests that PLA/PGA and PLLA/HA composite-based plates are both biocompatible for humans.

By providing direct comparative observations on the absorption patterns in the human body of absorbable plates frequently used for facial bone fracture surgery, the surgeon can predict the change in the absorbable plate to be used in patients. It can also help with the selection of absorbable plates, considering the degradation patterns, and with the facilitation of patient management.
Despite these interesting findings, there are a few limitations to our study. First, the number of patients who underwent secondary surgery to remove absorbable plates because of posttraumatic inflammatory responses was small; therefore, it is difficult to generalize our findings. Second, the numbers of patients who underwent surgery using PLLA/HA compositebased plates was smaller than that of patients receiving PLA/ PGA composite-based plates. Finally, the time elapsed from implant to removal of the absorbable plates was different among the patients in this study. Therefore, the possibility for uncontrolled bias exists when comparing these two groups. Long-term follow-up research and further evaluation of absorbable plate degradation patterns in humans should be performed to overcome these limitations.

\section{NOTES}

\section{Conflict of interest}

No potential conflict of interest relevant to this article was reported.

\section{Ethical approval}

The study was approved by the Institutional Review Board of Eulji Medical Center (IRB No. EMCIRB 19-19) and performed in accordance with the principles of the Declaration of Helsinki. Written informed consents were obtained.

\section{Patient consent}

The patients provided written informed consent for the publication and the use of their images.

\section{ORCID}

Young Min Kim https://orcid.org/0000-0001-5191-2593

Jong Hun Lee https://orcid.org/0000-0001-6417-6986

\section{REFERENCES}

1. Tripathi N, Goyal M, Mishra B, Dhasmana S. Zygomatic complex fracture: a comparative evaluation of stability using titanium and bio-resorbable plates as one point fixation. Natl J Maxillofac Surg 2013;4:181-7.

2. Al-Sukhun J, Lindqvist C. A comparative study of 2 implants used to repair inferior orbital wall bony defects: autogenous bone graft versus bioresorbable poly-L/DL-Lactide [P(L/DL) LA 70/30] plate. J Oral Maxillofac Surg 2006;64:1038-48.

3. Lionelli GT, Korentager RA. Biomechanical failure of metacarpal fracture resorbable plate fixation. Ann Plast Surg 2002;49: 202-6. 
4. Waris E, Ashammakhi N, Kaarela O, Raatikainen T, Vasenius J. Use of bioabsorbable osteofixation devices in the hand. J Hand Surg Br 2004;29:590-8.

5. Yerit KC, Hainich S, Turhani D, Klug C, Wittwer G, Ockher M, et al. Stability of biodegradable implants in treatment of mandibular fractures. Plast Reconstr Surg 2005;115:1863-70.

6. Uygur S, Cukurluoglu O, Ozmen S, Guclu TH, Sezgin B. Resorbable mesh plate in the treatment of blow-out fracture might cause gaze restriction. J Craniofac Surg 2009;20:71-2.

7. Bessho K, lizuka T, Murakami K. A bioabsorbable poly-L-lactide miniplate and screw system for osteosynthesis in oral and maxillofacial surgery. J Oral Maxillofac Surg 1997;55:941-5.

8. Rameshbabu N, Rao KP, Kumar TSS. Acclerated microwave processing of nanocrystalline hydroxyapatite. J Mater Sci 2005; 40:6319-23.

9. Shikinami Y, Okuno M. Bioresorbable devices made of forged composites of hydroxyapatite (HA) particles and poly-L-lactide (PLLA): Part I. basic characteristics. Biomaterials 1999;20:85977.

10. Shikinami Y, Matsusue Y, Nakamura T. The complete process of bioresorption and bone replacement using devices made of forged composites of raw hydroxyapatite particles/poly l-lactide (F-u-HA/PLLA). Biomaterials 2005;26:5542-51.

11. Goto K, Akiyama H, Kawanabe K, So K, Nakamura T. Use of HA-plla composite screws to fix acetabular bone graft in cemented THA: absorption pattern of screws in six patients. Key Eng Mater 2011;493:422-25.

12. Losken HW, van Aalst JA, Mooney MP, Godfrey VL, Burt T,
Teotia S, et al. Biodegradation of Inion fast-absorbing biodegradable plates and screws. J Craniofac Surg 2008;19:748-56.

13. Turvey TA, Bell RB, Tejera TJ, Proffit WR. The use of self-reinforced biodegradable bone plates and screws in orthognathic surgery. J Oral Maxillofac Surg 2002;60:59-65.

14. Bostman O, Pihlajamaki H. Clinical biocompatibility of biodegradable orthopaedic implants for internal fixation: a review. Biomaterials 2000;21:2615-21.

15. Choi HJ, Kim W, Youn S, Lee JH. Management of delayed infection after insertion of bioresorbable plates at the infraorbital rim. J Craniofac Surg 2012;23:524-5.

16. Middleton JC, Tipton AJ. Synthetic biodegradable polymers as orthopedic devices. Biomaterials 2000;21:2335-46.

17. Laughlin RM, Block MS, Wilk R, Malloy RB, Kent JN. Resorbable plates for the fixation of mandibular fractures: a prospective study. J Oral Maxillofac Surg 2007;65:89-96.

18. Landes CA, Ballon A, Roth C. In-patient versus in vitro degradation of P(L/DL)LA and PLGA. J Biomed Mater Res B Appl Biomater 2006;76:403-11.

19. Oonishi H, Hench LL, Wilson J, Sugihara F, Tsuji E, Kushitani S, et al. Comparative bone growth behavior in granules of bioceramic materials of various sizes. J Biomed Mater Res 1999;44: 31-43.

20. Furukawa T, Matsusue Y, Yasunaga T, Shikinami Y, Okuno M, Nakamura T. Biodegradation behavior of ultra-high-strength hydroxyapatite/poly (L-lactide) composite rods for internal fixation of bone fractures. Biomaterials 2000;21:889-98. 